\title{
Effects of Functional Footwear Designed for Decreasing Ground Reaction Force on Ankle and Foot Range of Motion During Gait in Healthy Individuals
}

\author{
Yong-Wook Kim, PhD, $\mathrm{PT}^{\dagger}$
}

Department of Physical Therapy, College of Medical Sciences, Jeonju University

Received: June 5, 2018 / Revised: June 8, 2018 / Accepted: June 23, 2018

(C) 2018 J Korean Soc Phys Med

\section{| Abstract |}

PURPOSE: This study was conducted to investigate the characteristics of a specific functional shoe in terms of the range of motion (ROM) of ankle and foot joints during walking when compared to a standardized shoe.

METHODS: Kinematic ROM data pertaining to ankle, tarsometatarsal, and metatarsophalangeal joints were collected from twenty-six healthy individuals during walking using a ten-camera motion analysis system. Kinematic ROM of each joint in three planes was obtained over ten walking trials consisting of two different shoe conditions. Visual3D motion analysis was finally used to coordinate the kinematic data. All kinematic ROM data were interpolated using a cubic spline algorithm and low-pass filtered with a cutoff frequency of $6 \mathrm{~Hz}$ for smoothing.

RESULTS: The overall ROM of the ankle joint in the sagittal and coronal planes when wearing the specific functional shoe was significantly decreased in both ankles

$\dagger$ Corresponding Author : Yong-wook Kim ptkim@jj.ac.kr, http://orcid.org/0000-0002-0311-9766

This is an Open Access article distributed under the terms of the Creative Commons Attribution Non-Commercial License (http://creativecommons.org/licenses/by-nc/3.0) which permits unrestricted non-commercial use, distribution, and reproduction in any medium, provided the original work is properly cited. during walking when compared to wearing a standard shoe $(p<.05)$. Significantly more flexibility was observed when wearing the specific functional shoe in the tarsometatarsal and metatarsophalangeal joints compared to a standard shoe $(p<.05)$.

CONCLUSION: Although clinical application of the specific functional shoe has shown clear positive effects on knee and ankle moments, the results of this study provide important background information regarding the kinematic mechanisms of these effects.

Key Words: Ankle, Foot, Functional shoe, Gait, Joint range of motion

\section{Introduction}

Maintaining normal alignment of the foot and ankle joints during gait is very important to the prevention of musculoskeletal problems of the lower extremity and increasing walking efficiency (Queen et al., 2011; Reilingh et al., 2010; Terrier et al., 2009). Specifically, abnormal alignment of the foot and ankle joints and segments in the stance phase during gait could result in various deformations such as pes cavus or planus and pronated or supinated rear foot (Boffeli and Waverly, 2016; Sawada et al., 2017). 
Over the past decade, specially-designed functional footwear for decreasing the ground reaction force has been developed as conservative treatment of lower extremity dysfunctions such as those affecting the knee, ankle, or foot joint (Radzimski et al., 2012; Shakoor et al., 2008; Shelburne et al., 2008; Terrier et al., 2009). Among these specialized functional shoes, a previous study reported that following use of the specific mobility footwear, the Flex-OA shoe, knee adduction moment was significantly reduced compared to when wearing general footwear (Shakoor et al., 2013). The anticipated positive results of specially-designed functional footwear are to improve gait function and motivation to participate in daily activities (Terrier et al., 2009). One of the characteristics of normal gait is bilateral symmetry of the left and right lower extremities in gait movements.

Because gait is primarily evaluated through observational analysis, the interpretations of gait analysis vary widely among clinicians with different expertise. For this reason, an objective and quantitative evaluation of gait movements would play an important role in verifying the effects of therapeutic interventions. Biomechanical three-dimensional analysis of gait movements in the laboratory is widely accepted as a quantitative method of gait evaluation.

Although specific functional shoes have been shown to significantly decrease knee and ankle joint loads, no studies has investigated whether this effect is universal or if it depends on characteristics of range of motion (ROM) of the ankle and foot caused by different shoe types during gait. Therefore, the purpose of this study was to verify differences in the ROM of the ankle and foot joints during walking when wearing a standard shoe and when wearing the specific mobility shoe.

\section{Methods}

\section{Participants}

This study recruited 26 healthy volunteers who met the selection criteria and consented to participate in the study. The study population consisted of eighteen males and eight females. Participants were given a detailed explanation of the study procedure and purpose and submitted written informed consent. This study was performed following approval from the Ethics Committee of the University of Central Lancashire (STEMH 347).

Participants who were able to walk freely for $10 \mathrm{~m}$ with an even gait were recruited for this study. Participants who had any neurological, musculoskeletal, or cardiopulmonary problems were excluded. The mean ages, heights, and weights of all participants were $28.7 \pm 10.3$ years, $176.8 \pm 9.1$ $\mathrm{cm}$, and $73.7 \pm 10.4 \mathrm{~kg}$, respectively. The $\mathrm{G}^{*}$ power 3.1 program was used to determine the appropriate sample size of study subjects to maintain an effect size of .8 , a power of .08 , and a significance level of .05 . The determined sample size was 21 , and 26 subjects were recruited for the study.

\section{Instrumentation and procedure}

Three-dimensional kinematic ROM data of ankle and foot joints were obtained using the Qualisys Motion Capture System (Qualisys, Gothenburg, Sweden) with ten Oqus-500 cameras while the participants walked along a $10 \mathrm{~m}$ walkway. To process the captured 3D marker coordinate data, Qualisys Track Manager software (Qualisys, Gothenburg, Sweden) was used at $100 \mathrm{~Hz}$. A $750 \mathrm{~mm}$ calibration wand was employed to calibrate the motion capture system and L-frame reference object identifying the lab origin.

Changes in the ankle and foot joint angles of the 26 participants were measured while walking when wearing the specific functional shoe (OA-Flex, DJO Global, Vista, CA, USA) and when wearing the standardized shoe using the Calibrated Anatomical System Technique. For the dynamic walking conditions, participants had 52 reflective markers $(14 \mathrm{~mm})$ bilaterally attached onto their rear feet, mid feet, fore feet, malleoli, femur epicondyles, greater 


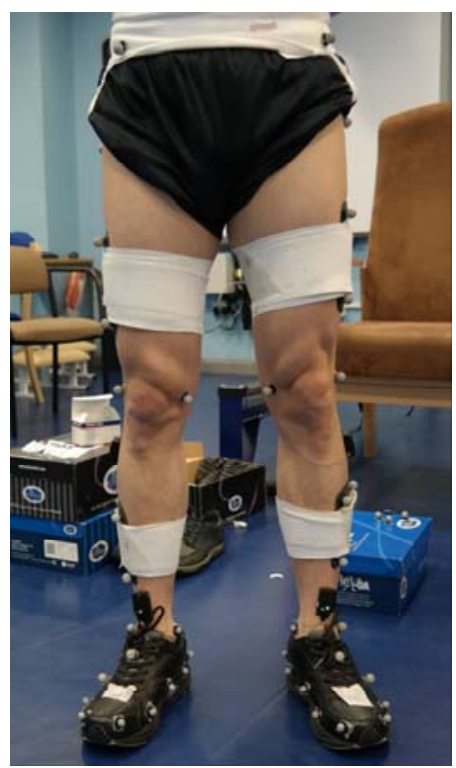

Fig. 1. Reflective marker sets on lower extremities for static calibration and dynamic motion capture

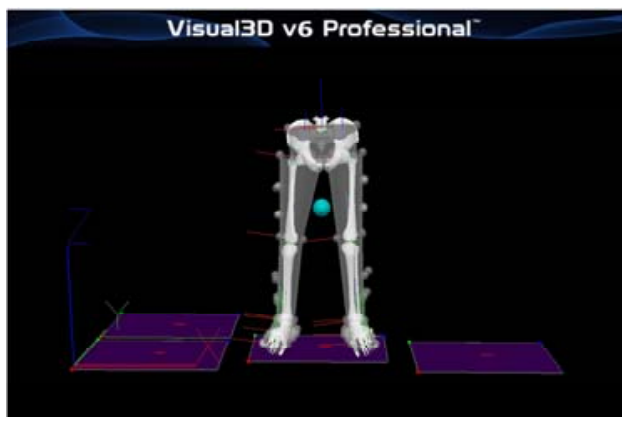

Fig. 2. Modified Oxford foot model with Calibrated Anatomical System Technique to measure range of motion of ankle and foot joints using a Visual3D program

trochanters, and anterior and posterior superior iliac spines (Fig. 1). Cluster Markers were attached bilaterally onto the shank and thigh according to the six-degrees-of-freedom (6DOF) model (Collins et al., 2009). First, a static calibration capture file was obtained from each participant to build the hybrid model to analyze dynamic trials. Following this static calibration capture, participants were asked to walk along a $10 \mathrm{~m}$ walkway in the laboratory at their preferred walking speed to obtain dynamic ROM

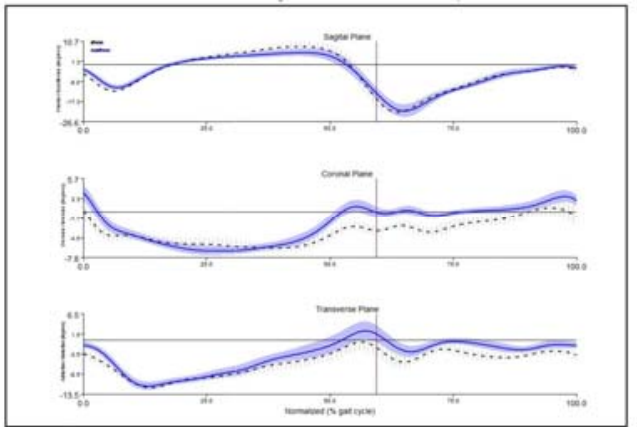

Fig. 3. Intra-individual variability of ankle joint motion during gait. Specific functional shoe angle (solid line) and standardized shoe angle (dot line) from 25 repetitive trials

data of the ankle and foot joints and segments. Participants executed a total of eight to ten walk trials. The order in which the shoes were worn (specific mobility shoe first or standard shoe first) was randomly assigned before the trials began.

Following the capture data collection, Visual3D motion analysis software (C-Motion, Rockville, MD, USA) was used to coordinate the data and report back. Ankle and foot ROM data were interpolated using a cubic spline algorithm and low-pass filtered with a cutoff frequency of $6 \mathrm{~Hz}$ for smoothing. The Visual3D created a visual representation of the bone segments in space that enabled the related joint angles to be calculated (Fig. 2). Examples of the mean ROM values and standard deviation bands of the ankle, tarsometatarsal, and metatarsophalangeal joints while walking are shown in Fig. 3 to Fig. 5. The $\mathrm{X}-\mathrm{Y}-\mathrm{Z}$ Cardan sequence defined the order of rotations following the Right-Hand Rule regarding the segment coordinate system. Joint kinematic data were normalized to the gait cycle starting with initial heel contact.

\section{Data analysis}

The SPSS ver. 23.0 statistical software was used for all analyses. Two-way repeated measures ANOVA with Bonferroni's correction was used to determine the effects of shoe conditions and left/right side differences. Statistical 


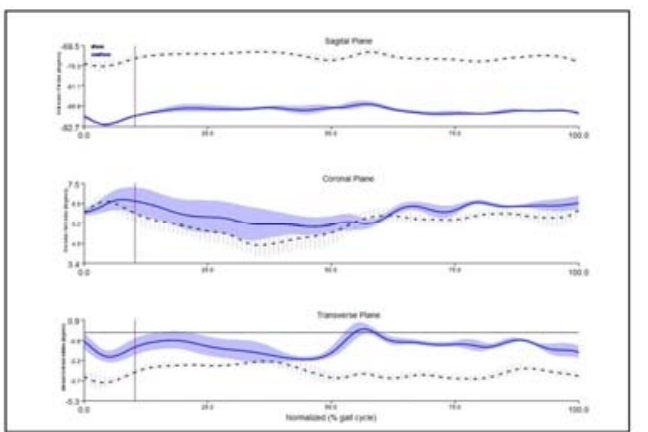

Fig. 4. Intra-individual variability of tarsometatarsal joint motion during gait. Specific functional shoe angle (solid line) and standardized shoe angle (dot line) from 25 repetitive trials

differences were defined as significant at the $\alpha=.05$ level.

\section{Results}

Data were found to be suitable for parametric testing.

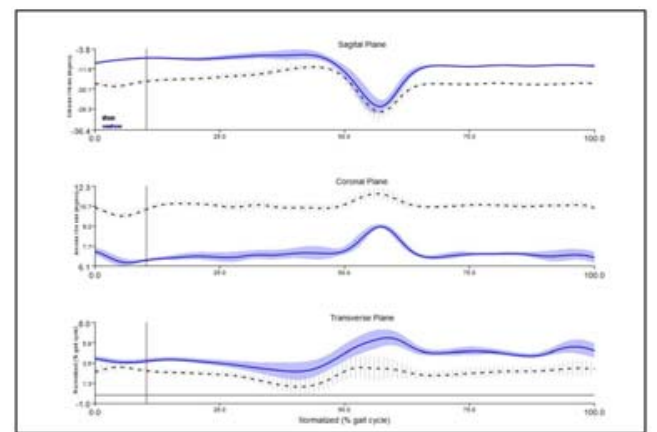

Fig. 5. Intra-individual variability of metatarsophalangeal joint motion during gait. Specific functional shoe angle (solid line) and standardized shoe angle (dot line) from 25 repetitive trials

A two factor repeated measures ANOVA with Bonferroni's correction was used to determine the effects of shoe condition and ankle and foot side during gait. Significant differences were observed in ankle ROMs between shoe conditions in the stance and swing phases during gait (Table 1).

Table 1. Repeated Measures ANOVA Comparing Ranges of Ankle Joint under the Shoe Conditions and Ankle Sides During Walking $(\mathrm{N}=26)$

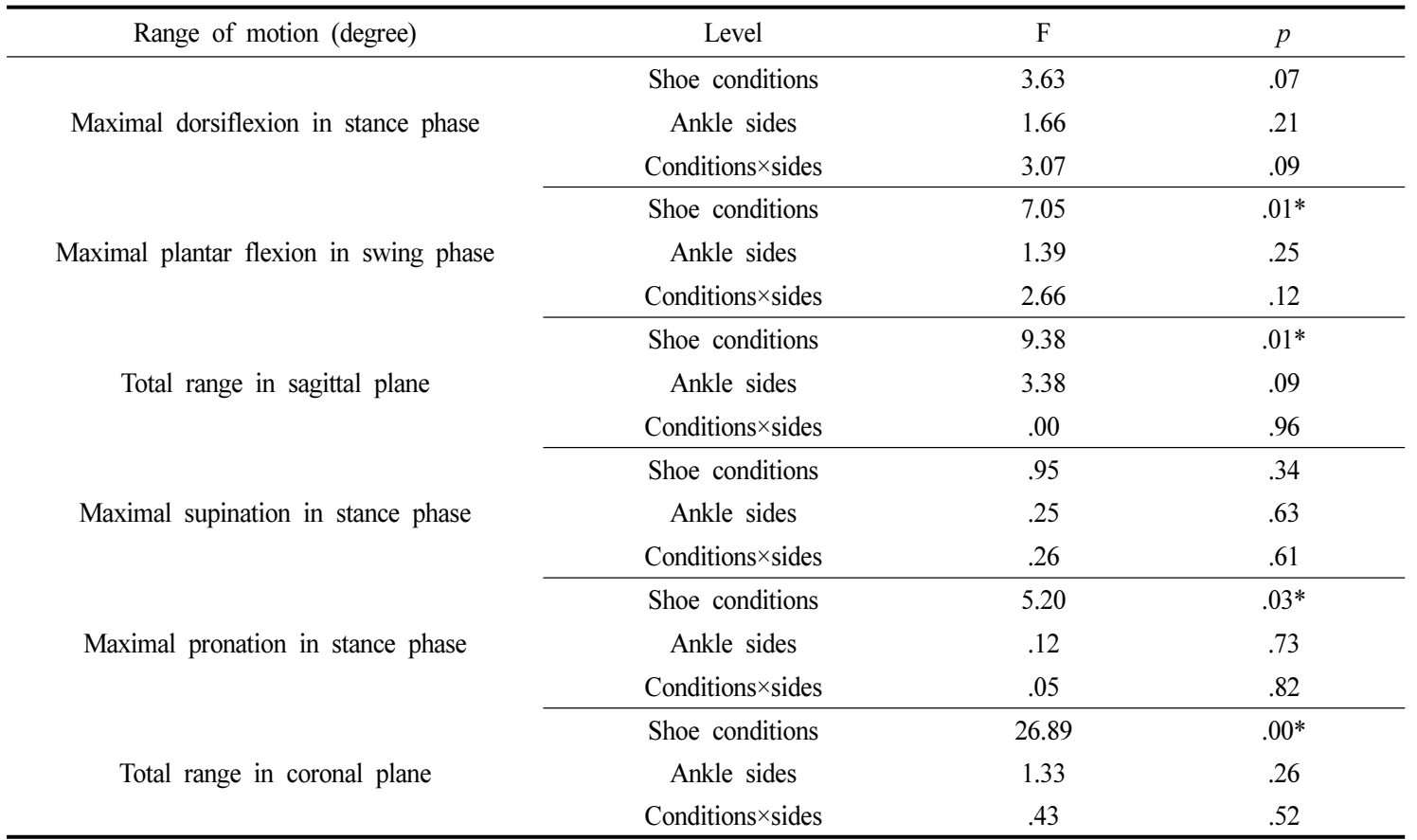

$* p<.05$ 
Table 2. Repeated Measure ANOVA Comparing Ranges of Tarsometatarsal Joint under the Shoe Conditions and Foot Sides During Walking $(\mathrm{N}=26)$

\begin{tabular}{cccc}
\hline Range of motion (degree) & Level & F & $p$ \\
\hline \multirow{3}{*}{ Total range in sagittal plane } & Shoe conditions & .00 & .99 \\
& Foot sides & .40 & .53 \\
& Conditions $\times$ sides & .06 & $.04^{*}$ \\
\cline { 2 - 4 } Total range in coronal plane & Shoe conditions & 4.30 & .36 \\
& Foot sides & .88 & .30 \\
\cline { 2 - 4 } Total range in transverse plane & Conditions $\times$ sides & 1.11 & $.03^{*}$ \\
& Shoe conditions & 5.28 & .35 \\
\hline
\end{tabular}

${ }^{*} p<.05$

During walking in the specific functional shoe condition, the ROM of maximal plantar flexion in the swing phase $(60-100 \%$ of gait cycle; $\mathrm{F}(1,25)=7.05 ; p<.05)$ and the total ankle ROM in the sagittal plane $(\mathrm{F}(1,25)=3.37 ; p<.05)$ were both significantly decreased in both ankles (Table 1). In addition, there were significant differences in the ROM of maximal ankle pronation in the stance phase ( $\mathrm{F}$ $(1,25)=5.20 ; p<.05)$ and the total ROM of the ankle joint in the coronal plane $(\mathrm{F}(1,25)=26.89 ; p<.05)$ between shoe conditions during walking (Table 1). However, there were no interaction effects of shoe conditions and ankle sides in any ROM values of the ankle joint ( $p>.05$ ) (Table 1).

Significantly more flexibility was observed during walking in the tarsometatarsal joint when wearing the specific functional shoe compared to when wearing the standardized shoe in the coronal $(\mathrm{F}(1,25)=4.30 ; p<.05)$ and transverse planes $(\mathrm{F}(1,25)=5.28 ; p<.05)$ (Table 2). In the sagittal plane, the total ROM of the tarsometatarsal joint did not differ significantly between shoe conditions during walking ( $p>.05)$ (Table 2). In addition, there were no interaction effects of shoe conditions and foot sides in all ROM values of the tarsometatarsal joint $(p>.05)$ (Table 2).

Although the maximum extension range of the metatarsophalangeal joint of the sagittal plane in the stance phase $(60-100 \%$ of gait cycle) did not significantly differ between shoe conditions $(p>.05)$, wearing the specific functional shoe did significantly increase the range of maximal flexion $(\mathrm{F}(1,25)=7.03 ; p<.05)$ and total $\mathrm{ROM}$ of the sagittal plane $(\mathrm{F}(1,25)=33.63 ; p<.05)$ in both feet during walking (Table 3 ). In addition, significantly more flexibility was observed in the metatarsophalangeal joint when wearing the specific functional shoe compared to when wearing the standardized shoe in the coronal $(\mathrm{F}(1,25)$ $=4.46 ; p<.05)$ and transverse planes $(\mathrm{F}(1,25)=14.20 ; p<.05)$ during walking (Table 3). However, there were no interaction effects of shoe conditions and foot sides in any ROM values of the metatarsophalangeal joint $(p>.05)$ (Table 3).

\section{Discussion}

This study was conducted to investigate the effects of the specific functional shoe, which has been shown to have significant effects on musculoskeletal disorders of the lower extremities by reducing knee and ankle joint moments and ground reaction forces on the ROM of the ankle and foot joints and segments during walking, based on comparison 
Table 3. Repeated Measures ANOVA Comparing Ranges of Metatarsophalangeal Joint under the Shoe Conditions and both Sides During Walking $(\mathrm{N}=26)$

\begin{tabular}{|c|c|c|c|}
\hline Range of motion (degree) & Level & $\mathrm{F}$ & $p$ \\
\hline \multirow{3}{*}{ Maximal flexion in stance phase } & Shoe conditions & 7.03 & $.01^{*}$ \\
\hline & Foot sides & 1.87 & .18 \\
\hline & Conditions $\times$ sides & .04 & .85 \\
\hline \multirow{3}{*}{ Maximal extension in stance phase } & Shoe conditions & .28 & .60 \\
\hline & Foot sides & 1.71 & .20 \\
\hline & Conditions $\times$ sides & .00 & .99 \\
\hline \multirow{3}{*}{ Total range in sagittal plane } & Shoe conditions & 33.63 & $.00 *$ \\
\hline & Foot sides & .05 & .83 \\
\hline & Conditions $\times$ sides & .11 & .74 \\
\hline \multirow{3}{*}{ Total range in coronal plane } & Shoe conditions & 4.46 & $.04 *$ \\
\hline & Foot sides & .91 & .35 \\
\hline & Conditions $\times$ sides & .95 & .34 \\
\hline \multirow{3}{*}{ Total range in transverse plane } & Shoe conditions & 14.20 & $.00 *$ \\
\hline & Foot sides & 3.99 & .06 \\
\hline & Conditions $\times$ sides & .00 & .98 \\
\hline
\end{tabular}

${ }^{*} p<.05$

to the effects of a standardized shoe (Erhart-Hledik et al., 2012; Radzimski et al., 2012; Shakoor et al., 2008; Shakoor et al., 2013). This is the first attempt in Korea to analyze and compare the ROM of real-time motion of ankle and foot segments through 3D motion analysis using an infrared camera system during walking when wearing two different shoes (Fig. 3, 4, 5). This is because it is not easy to analyze real-time movement of the ankle and foot during walking since the foot complex is composed of seven tarsal bones, five metatarsal bones, and fourteen phalangeal bones, as well as many small joints and segments. In this study, the Modified Oxford Foot model developed by the Institute of Biomechanical Motion Analysis (University of Central Lancashire, Preston, UK) and Visual3D motion analysis program were used to divide the foot into forefoot, midfoot, and rearfoot segments for 3D motion analysis (Kim, 2018; Kim et al., 2018).

The results revealed that the ROM of the maximal dorsiflexion in the stance phase did not differ significantly between shoe conditions during gait. However, the ROM of the maximal plantar flexion in the swing phase and the total ankle ROM in the sagittal plane were significantly decreased when wearing the specific functional shoe in both ankles during walking $(p<.05)$. In addition, the total $\mathrm{ROM}$ and maximal pronation range in the coronal plane were significantly decreased when wearing the specific function shoe, similar to the effect seen in the ankle motion of the sagittal plane. Previous studies using 3D gait analysis have primarily analyzed spatial-temporal gait variables such as walking speed, step length, stance time, stride length, single-support time, double support time (McNair et al., 2018; Queen et al., 2011), or the general angles of the hip, knee, and ankle joints during gait (Kim and Kim, 2014; Mannering et al., 2017). Although there are no previous studies with which these results can be directly compared to, Sinclair et al. (2015) reported that, in healthy individuals, 
barefoot running was linked with significantly more plantarflexion and total ROM of the ankle joint than shod running. The results of the present study differed from those of the previous study in terms of the ROM of the ankle joint, likely because there were differences in the shoe condition, measurement condition, and participant characteristics.

In contrast to the results of the ankle joint ROMs, significantly more motion was observed during walking in the coronal and transverse planes in the tarsometatarsal joint when wearing the specific functional shoe than when wearing the standardized shoe, although this was not the case in the sagittal plane $(p<.05)$. The lack of significant differences in the ROMs of the tarsometatarsal joint between the two shoe conditions in the sagittal plane may have occurred because the tarsometatarsal joint is more active in the coronal plane, such as during adduction and abduction. Similar to the results of the tarsometatarsal joint motion, ( $p>.05)$, the range of maximal flexion ( $\mathrm{F}=7.03$; $p<.05)$ and total ROM of the metatarsophalangeal joint in the sagittal plane when wearing the specific functional shoe increased significantly in both feet during walking, while maximal extension did not differ significantly between shoe conditions.

The results of this study show that the use of the special functional shoe reduces the ROM of the ankle joint and increases movement of the tarsometatarsal and metatarsophalangeal joints when compared to use of the standardized shoe. These results are similar to those of previous studies in which those who walked barefoot showed more movement of the foot joints than those who wore shoes. Therefore, the results of this study help explain the results of previous studies in that the use of the specific functional shoe effectively decreased knee moments and ground reaction force (Radzimski et al., 2012; Shakoor et al., 2008).

It should be noted that there are some limitations to this study. Specifically, the study was conducted on healthy individuals, who were mostly younger than the general age range of those suffering from musculoskeletal problems such as knee and ankle osteoarthritis. Although clinical application of the specific functional shoe has shown clearly positive effects in knee and ankle moments, the results presented herein provide important information that will be useful to future research on the moment effect when using the specific functional shoe. Therefore, further studies should be conducted to develop a variety of highly flexible special shoes to examine the effects of these biomechanical variables in patients with lower extremity osteoarthritis.

\section{Conclusion}

The purpose of this study was to investigate the effects of the specific functional shoe on ROM of the ankle and foot joints compared to a standard shoe during walking in twenty-six healthy male and female adults. According to the results of this study, there were significantly increased differences in most ROM values of foot joints when wearing the specific functional shoe. These results suggest that application of a highly flexible shoe to various musculoskeletal disorders of the lower extremities, such as knee and ankle osteoarthritis, might facilitate symptom relief.

\section{References}

Boffeli TJ, Waverly BJ. Angle and Base of Gait Long Leg Axial and Intraoperative Simulated Weightbearing Long Leg Axial Imaging to Capture True Frontal Plane Tibia to Calcaneus Alignment in Valgus and Varus Deformities of the Rearfoot and Ankle. J Foot Ankle Surg. 2016;55(5):1043-51.

Collins TD, Ghoussayni SN, Ewins DJ, et al. A six degrees-of-freedom marker set for gait analysis: repeatability and comparison with a modified Helen Hayes set. Gait Posture. 2009;30(2):173-80. 
Erhart-Hledik JC, Elspas B, Giori NJ, et al. Effect of variable-stiffness walking shoes on knee adduction moment, pain, and function in subjects with medial compartment knee osteoarthritis after 1 year. J Orthop Res. 2012;30(4):514-21.

Kim WS, Kim EY. Comparing self-selected speed walking of the elderly with self-selected slow, moderate, and fast speed walking of young adults. Ann Rehabil Med. 2014;38(1):101-8.

Kim Y, Richards J, Lidtke RH, et al. Characteristics of clinical measurements between biomechanical responders and non-responders to a shoe designed for knee osteoarthritis. Gait Posture. 2018;59:23-7.

Kim Y. Concurrent validity and clinical usefulness of universal plastic goniometer for hip internal and external rotation range measurement. J Korean Soc Phys Med. 2018;13(1):99-105.

Mannering N, Young T, Spelman T, et al. Three-dimensional knee kinematic analysis during treadmill gait: Slow imposed speed versus normal self-selected speed. Bone Joint Res. 2017;6(8):514-21.

McNair PJ, Boocock MG, Dominick ND, et al. A Comparison of Walking Gait Following Mechanical and Kinematic Alignment in Total Knee Joint Replacement. J Arthroplasty. 2018;33(2):560-64.

Queen RM, Carter JE, Adams SB, et al. Coronal plane ankle alignment, gait, and end-stage ankle osteoarthritis. Osteoarthritis Cartilage. 2011;122(11):1338-42.

Radzimski AO, Mündermann A, Sole G. Effect of footwear on the external knee adduction moment - A systematic review. Knee. 2012;19(3):163-75.

Reilingh ML, Beimers L, Tuijthof GJ, et al. Measuring hindfoot alignment radiographically: the long axial view is more reliable than the hindfoot alignment view. Skeletal Radiol. 2010;39(11):1103-8.

Sawada T, Tanimoto K, Tokuda K, et al. Rear foot kinematics when wearing lateral wedge insoles and foot alignment influence the effect of knee adduction moment for medial knee osteoarthritis. Gait Posture. 2017;57:177-81.

Shakoor N, Lidtke RH, Sengupta M, et al. Effects of specialized footwear on joint loads in osteoarthritis of the knee. Arthritis Rheum. 2008;59(9):1214-20.

Shakoor N, Lidtke RH, Wimmer MA, et al. Improvement in knee loading after use of specialized footwear for knee osteoarthritis: results of a six-month pilot investigation. Arthritis Rheum. 2013;65(5):1282-9.

Shelburne KB, Torry MR, Steadman JR, et al. Effects of foot orthoses and valgus bracing on the knee adduction moment and medial joint load during gait. Clin Biomech (Bristol, Avon). 2008;23(6):814-21.

Sinclair J, Atkins S, Richards J, et al. Modelling of Muscle Force Distributions During Barefoot and Shod Running. J Hum Kinet. 2015;47:9-17.

Terrier P, Dériaz O, Meichtry A, et al. Prescription footwear for severe injuries of foot and ankle: effect on regularity and symmetry of the gait assessed by trunk accelerometry. Gait Posture. 2009;30(4):492-6. 